\section{Strongly interacting one-dimensional Bose condensates in power law potentials}

To cite this article: B. Tanatar 2000 EPL 51261

View the article online for updates and enhancements.
Related content

- The ground state and vortices in a two-
$\frac{\text { dimensional Bose gas confined in a }}{\text { harmonic trap }}$
B Tanatar
- A density-functional approach to
$\frac{\text { fermionization in the 1D Bose gas }}{\text { Joachim Brand }}$
- Low-energy properties of a one-
$\frac{\text { dimensional system of interacting bosons }}{\text { with boundaries }}$
M. A. Cazalilla

Recent citations
Diverging probability-density functions for
flat-top solitary waves
Avner Peleg et al
Nonlinear waves in Bose-Einstein condensates: physical relevance and mathematical techniques
R Carretero-González et al
- Dynamics of shallow dark solitons in a trapped gas of impenetrable bosons N. P. Proukakis et al 
Europhys. Lett., 51 (3), pp. 261-267 (2000)

\title{
Strongly interacting one-dimensional Bose condensates in power law potentials
}

\author{
B. TANATAR \\ Department of Physics, Bilkent University - Bilkent, 06533 Ankara, Turkey
}

(received 10 January 2000; accepted in final form 31 May 2000)

PACS. 03.75.Fi - Phase coherent atomic ensembles; quantum condensation phenomena.

PACS. 05.30.Jp - Boson systems.

PACS. 32.80.Pj - Optical cooling of atoms; trapping.

\begin{abstract}
We study the interaction effects on the condensates by considering a model of one-dimensional bosons. The power law type external potential allows for the formation of a condensate in these systems. Using a density-functional theory type formalism we obtain an equation describing the condensate wave function in the limit of very strong interactions between the bosons. The properties of the condensate in the model system with strong interactions are investigated. The equivalence of strongly interacting bosons to noninteracting spinless fermions is demonstrated.
\end{abstract}

The observation of Bose-Einstein condensation (BEC) phenomena in externally confined atomic vapors [1] has had a big impetus on the theoretical study of boson systems in general. The thermodynamic, ground-state static and dynamic properties of condensates are thoroughly reviewed [2]. As the number of atoms in the condensates increases the deviations from the weak-coupling description will be more and more important, and this fact has been recognized earlier on $[3,4]$ and emphasized recently $[5,6]$.

The aim of this work is to explore the properties of one-dimensional (1D) boson condensates in power law external potentials [7]. The power law trapping potentials are not only of academic interest, but are thought to be of relevance to adiabatically cooled systems [8] to achieve BEC. We investigate the dependence on boson number $N$ and more interestingly the dependence on short-range interaction strength of the condensate wave function at zero temperature. There are several motivations for undertaking such a study. Firstly, in highly anisotropic trap potentials as used in the present experiments, the so-called cigar-shaped condensates are formed which may be modeled by a 1D equation [9]. Secondly, the role of interaction effects may be studied in a model system where an exact solution to the homogeneous problem is known. We use the local-density approximation to describe the interaction effects on the condensate and obtain a new equation valid in the strong-interaction limit.

The 1D bosons have attracted considerable attention in recent years [10]. Pearson, Pang and Chen [11] studied the interacting Bose gas in 1D subject to power law potentials employing the path-integral Monte Carlo method. They found that a macroscopically large number of particles occupy the lowest single-particle state in a finite system of hard-core bosons at some 
critical temperature. The validity of the Gross-Pitaevskii [12] (GP) equation even at zero temperature when the interactions are strong is an important question in our understanding of the boson condensates. A rigorous derivation of the GP equation has been provided for 3D and 2D Bose gases [13]. To set the stage, in the following we first discuss the GP equation description of $1 \mathrm{D}$ systems in power law trapping potentials. We next introduce the correction terms to obtain the modified GP equation. Lastly, we specialize to the infinite coupling strength limit and study some of the properties of strongly coupled condensates.

We consider a system of interacting bosons in $1 \mathrm{D}$ confined in a power law potential $V_{\text {ext }}(x)=V_{0}(|x| / L)^{\eta}$, where $V_{0}$ and $L$ are some suitable energy and length parameters defining the external potential, and $\eta<2$ controls the confinement strength [7]. For a weakly interacting system the condensate wave function $\psi(x)$ satisfies the Gross-Pitaevskii [12] (GP) equation

$$
\left\{-\frac{1}{2} \frac{\mathrm{d}^{2}}{\mathrm{~d} x^{2}}+|x|^{\eta}+g N|\psi|^{2}\right\} \psi=\mu \psi,
$$

in which we have scaled the lengths by $a=\hbar /\left(m V_{0}\right)^{1 / 2}$ and energies by $V_{0}$, and have taken $L / a=1$ for convenience. Our dimensionless condensate wave function is defined as $\psi=$ $(a / N)^{1 / 2} \phi$. The dimensionless quantity $g$ describes the coupling strength of boson-boson interaction. For the model of $1 \mathrm{D}$ bosons interacting through a delta-function potential of strength $C$ as discussed by Lieb and Liniger [14] we have $g=C /\left(V_{0} a\right)$. The above equation can be obtained from the minimization of the energy functional

$$
E / N=\int_{-\infty}^{\infty} \mathrm{d} x\left\{\frac{1}{2}\left|\frac{\mathrm{d} \psi}{\mathrm{d} x}\right|^{2}+|x|^{\eta}|\psi|^{2}+\frac{g}{2} N|\psi|^{4}\right\},
$$

subject to the normalization condition, which defines the chemical potential $\mu$ in eq. (1). We obtain the condensate wave function $\psi$ for different $N$ values by numerically minimizing the energy functional as shown in fig. 1, along with the Thomas-Fermi approximation results obtained by neglecting the kinetic-energy term. We find that a condensate cloud of a certain size, which depends on $g$ and $N$, exists because of the external potential. For a given confining potential characterized by $\eta$, the interaction strength $g$ and particle number $N$ enter only through the combination $g N$, so the curves in fig. 1 may be read as indicating either fixed $g$ and varying $N$ or vice versa. Using the argument [15] that the balance between the interaction energy $g N / R$ and the confinement energy $V_{0} R^{\eta}$ determine the size of the condensate, we estimate it to be $R / a=(g N)^{1 /(\eta+1)}$ which is in good agreement with the numerical results shown in fig. 1.

It was argued by Nunes [6] that a generalization of the GP equation may be developed in the spirit of the Kohn-Sham theory [16] by making the local-density approximation. This improves the mean-field GP equation by incorporating the correlations between the particles. Assuming that the full ground-state energy per particle for the homogeneous problem is known, the functional

$$
E / N=\int_{-\infty}^{\infty} \mathrm{d} x\left\{\frac{1}{2}\left|\frac{\mathrm{d} \psi}{\mathrm{d} x}\right|^{2}+|x|^{\eta}|\psi|^{2}+\epsilon(\rho)|\psi|^{2}\right\}
$$

is minimized to yield

$$
\left\{-\frac{1}{2} \frac{\mathrm{d}^{2}}{\mathrm{~d} x^{2}}+|x|^{\eta}+\frac{\partial(\epsilon \rho)}{\partial \rho}\right\} \psi=\mu \psi,
$$

with $\rho=N|\psi|^{2}$, and $\epsilon(\rho)=E(\rho) / N$ is the ground-state energy per particle of the homogeneous system. Several authors [4-6] made use of the perturbation theory expansion [17] 


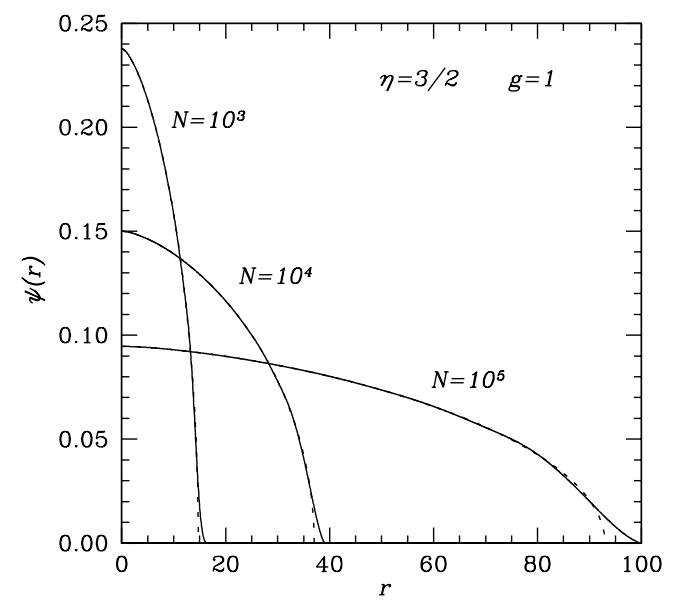

Fig. 1

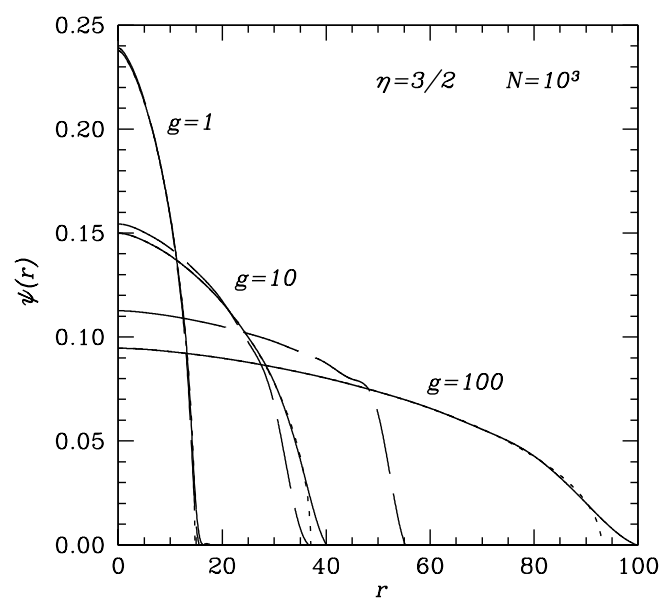

Fig. 2

Fig. 1 - The condensate wave function calculated with the Gross-Pitaevskii equation (solid lines) and the corresponding Thomas-Fermi approximation (dotted lines), for $N=10^{3}, N=10^{4}$, and $N=10^{5}$ particles. The power law potential exponent is $\eta=3 / 2$ and the interaction strength is $g=1$.

Fig. 2 - Comparison of the condensate wave function in the Gross-Pitaevskii (solid lines) and modified Gross-Pitaevskii (dashed lines) approaches, for $g=1, g=10$, and $g=100$. The number of particles is $N=10^{3}$, and dotted lines indicate the Thomas-Fermi approximation to the Gross-Pitaevskii results.

in $3 \mathrm{D}$ to calculate the corrections to the GP equation. For the $1 \mathrm{D}$ bosons interacting via a short-range delta-function potential the ground-state energy is given by [18] $\epsilon(\rho)=g \rho / 2-$ $2 g^{3 / 2} \rho^{1 / 2} /(3 \pi)+\ldots$ In contrast to the perturbation expansion in 3D uniform Bose systems [17], the series in 1D has terms with alternating signs. Thus, the modified GP equation (with next-order correction) becomes

$$
\left\{-\frac{1}{2} \frac{\mathrm{d}^{2}}{\mathrm{~d} x^{2}}+|x|^{\eta}+g N|\psi|^{2}-\frac{g^{3 / 2}}{\pi} N^{1 / 2} \psi\right\} \psi=\mu \psi
$$

with the corresponding modified GP functional

$$
E / N=\int_{-\infty}^{\infty} \mathrm{d} x\left\{\frac{1}{2}\left|\frac{\mathrm{d} \psi}{\mathrm{d} x}\right|^{2}+|x|^{\eta}|\psi|^{2}+\frac{g}{2} N|\psi|^{4}-\frac{2 g^{3 / 2}}{3 \pi} N^{1 / 2}|\psi|^{2} \psi\right\}
$$

The results of our calculations for various coupling strengths within the modified GP equation are shown in fig. 2. At fixed number of particles $\left(N=10^{3}\right)$, we observe deviations from the GP equation results in two respects. Firstly, the $\psi(x)$ obtained in the modified GP approach is larger than that in the GP approach for small $x$. This is in contrast to the situation in 3D studied before [4-6] where the modified GP equation yields lower values for $\psi$. The reason for this behavior is in the attractive and repulsive nature of the corrections for the low-density expansions of homogeneous energy density in 1D and 3D, respectively. Secondly, for large values of $x$ up to the edge of the condensate, $\psi(x)$ in the modified GP scheme falls well below the result of the GP equation. These deviations at large $x$, which again can be traced to the attractive contribution to the energy density, become quite sizeable as the interaction strength $g$ increases, and perhaps indicate the insufficiency of the number of terms required 
in the low-density expansion of $\epsilon(\rho)$. We have checked this by including higher-order terms in the perturbation series and found improvement.

We now turn to the limit of very strong coupling between the interacting bosons. The homogeneous problem was solved by Lieb and Liniger [14] and the energy density as a function of the coupling strength $g$ is provided as a series expansion [18]. For large coupling strengths the energy density is given by $\epsilon=\left(\pi^{2} / 6\right) g^{2} /(g / \rho+2)^{2}$, and in particular, when $g$ is infinite, it simply reduces to $\epsilon=\pi^{2} \rho^{2} / 6$. Using the above Kohn-Sham form of the equation of motion we obtain

$$
\left\{-\frac{1}{2} \frac{\mathrm{d}^{2}}{\mathrm{~d} x^{2}}+|x|^{\eta}+\frac{\pi^{2}}{2} N^{2}|\psi|^{4}\right\} \psi=\mu \psi,
$$

for the condensate wave function in the strongly coupled limit. Interestingly, the new nonlinear equation contains a fifth-order term with a coefficient proportional to $N^{2}$, and the cubic term is altogether missing. The strong-coupling equation for $\psi$ can be obtained from the minimization of the functional

$$
E / N=\int_{-\infty}^{\infty} \mathrm{d} x\left\{\frac{1}{2}\left|\frac{\mathrm{d} \psi}{\mathrm{d} x}\right|^{2}+|x|^{\eta}|\psi|^{2}+\frac{\pi^{2}}{6} N^{2}|\psi|^{6}\right\} .
$$

Similarly to the cubic GP equation, we develop a Thomas-Fermi type approximation for eq. (7), neglecting the kinetic-energy term. The Thomas-Fermi (TF) approximation in the strong-coupling limit gives for the condensate wave function

$$
\psi_{\mathrm{TF}}^{4}=\frac{2}{\pi^{2} N^{2}}\left(\mu-|x|^{\eta}\right) \theta\left(\mu-|x|^{\eta}\right),
$$

where the chemical potential is determined by

$$
\frac{\pi N}{2 \sqrt{2}}=\int_{0}^{\mu^{1 / \eta}} \mathrm{d} x\left(\mu-x^{\eta}\right)^{1 / 2} .
$$

It is interesting at this point to note that according to Girardeau's theorem [19] an infinitely strong repulsion between bosons in one-dimension effectively turns the particles into noninteracting spinless fermions as demonstrated by Lieb and Liniger [14]. We apply the straightforward Thomas-Fermi approximation [20] to a system of noninteracting spinless fermions in a power law trapping potential, defining the density $n(x)=k_{\mathrm{F}}(x) / \pi$, in terms of the "local" Fermi wave number. Solving the TF equation

$$
\frac{\hbar^{2} k_{\mathrm{F}}^{2}(x)}{2 m}+V_{0} \frac{|x|^{\eta}}{L^{\eta}}=E_{\mathrm{F}},
$$

we obtain for the fermion density at zero temperature

$$
n(x)=\frac{\sqrt{2}}{\pi}\left(E_{\mathrm{F}}-|x|^{\eta}\right)^{1 / 2} \theta\left(E_{\mathrm{F}}-|x|^{\eta}\right)
$$

(in our reduced units), where the Fermi energy is given by

$$
E_{\mathrm{F}}=\left[\frac{2 \eta}{2+\eta} \frac{N}{\sqrt{2} F(\eta)}\right]^{2 \eta /(2+\eta)},
$$

in which $F(\eta)=\int_{0}^{1} \mathrm{~d} y y^{1 / \eta-1} / \sqrt{1-y}$ is a numerical factor stemming from the semi-classical density of states for the power law potential. Comparing with eq. (9), we observe that Girardeau's theorem is exactly satisfied (at the TF level) for infinitely strongly coupled 1D bosons in an external potential. 


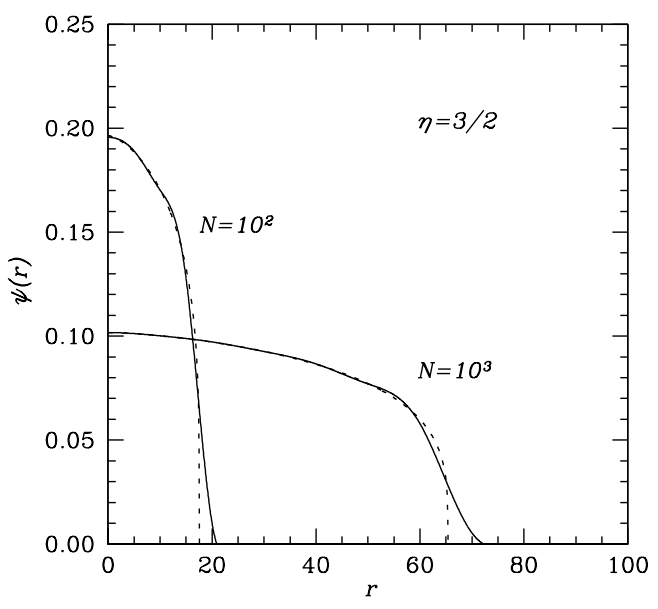

Fig. 3

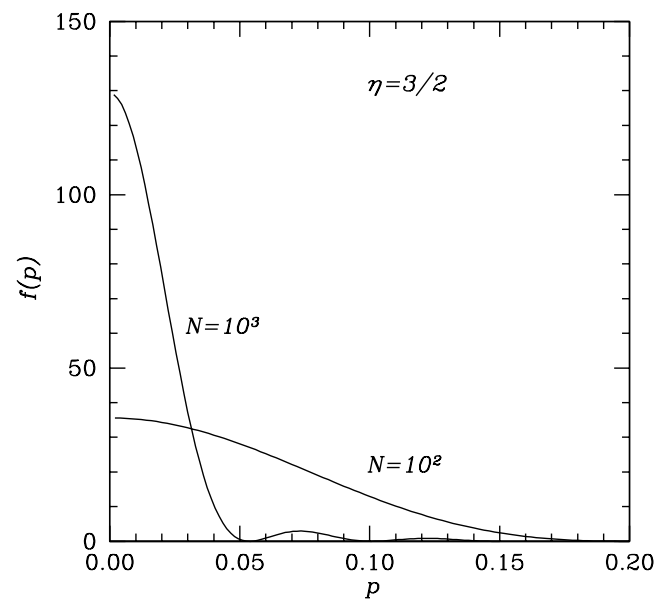

Fig. 4

Fig. 3 - The condensate wave function (solid lines) in the infinite-coupling limit, for $N=10^{2}$ and $N=10^{3}$ particles. The corresponding Thomas-Fermi results are indicated by dotted lines.

Fig. 4 - The momentum distribution of condensed bosons in the strong-coupling limit for $N=10^{2}$ and $N=10^{3}$ particles.

In fig. 3 we display the numerical solution of eq. (7) along with the TF approximation results (eqs. (9) and (10)) for the condensate wave function in the strong-coupling limit. Remarkably, a condensate seems to exist in the sense that a finite cloud extends over a finite length. It is interesting to note that the solution of eq. (5) is rather similar to the result of $\psi(x)$ we obtain within the modified GP equation, say for $g=100$ (cf. fig. 2). The corresponding TF approximation to eq. (7) is also plotted in fig. 3, and we observe that it is quite accurate for small $x$. We have checked numerically that TF expressions of the density distribution for the strongly interacting bosons and noninteracting spinless fermions are identical in accordance with Girardeau's theorem. The momentum distribution of condensed bosons in the confining potential $f(p)=4\left|\int_{0}^{\infty} \mathrm{d} x \cos (p x) \psi(x)\right|^{2}$, where $p$ is the particle momentum, is shown in fig. 4 , for $N=10^{2}$ and $N=10^{3}$ particles. We also remark that the infinite-coupling expressions developed above may be systematically improved by including more terms for large $g$ values.

The strong-coupling effects in condensed Bose systems have been addressed by a number of authors. Ziegler and Shukla [21] using the slave-boson technique found that the effective potential grows only linearly in the asymptotic regime in contrast to the $\psi^{4}$ behavior in the GP functional. Thus, the condensate becomes weaker and is easily destroyed by fluctuation effects. Cherny and Shanenko [22] proposed a strong-coupling generalization of the Bogoliubov model. Our results indicate that a condensate exists even for an infinitely strongly interacting 1D model system. It is interesting to note that recently Gammal et al. [23] have found a $\psi^{5}$ correction term to the GP equation by including three-body scattering effects in the $3 \mathrm{D}$ inhomogeneous systems. It would also be interesting to check our results for strongly interacting bosons with a short-range potential in 1D (also under an external potential) by Monte Carlo calculations to see if the new type of nonlinear Schrödinger equation correctly describes the properties of the condensate. Recent calculations by Giorgini, Boronat, and Casulleras [24] could easily be extended to study the system considered here. 
Finally, we comment on the collective modes of the strongly interacting 1D Bose condensates. Because of the $\psi^{5}$ nonlinearity and the relation between the chemical potential $\mu$ and density $\rho$ within the corresponding Thomas-Fermi approximation, rather different kinds of collective modes are expected. Applying the hydrodynamic approach employed for condensates satisfied by the GP equation [25] we also obtain $\omega^{2} \delta \rho+\frac{\mathrm{d}}{\mathrm{d} x}\left(\rho_{\mathrm{TF}} \frac{\mathrm{d}}{\mathrm{d} x} \delta \mu\right)=0$, in which $\delta \mu=\pi^{2} N^{2} \rho_{\mathrm{TF}} \delta \rho, \rho_{\mathrm{TF}}=\psi_{\mathrm{TF}}^{2}$, and $\delta \rho=\rho-\rho_{\mathrm{TF}}$. Thus, the collective mode equation becomes

$$
\omega^{2} \delta \rho+\pi^{2} N^{2} \frac{\mathrm{d}}{\mathrm{d} x}\left(\rho_{\mathrm{TF}} \frac{\mathrm{d}}{\mathrm{d} x}\left(\rho_{\mathrm{TF}} \delta \rho\right)\right)=0
$$

describing excitations of a strongly interacting system. For arbitrary $\eta$ of power law potential exponent the above equation has to be solved numerically.

In this work we have used power law potentials with exponent $\eta<2$, for which the existence of a BEC (in a noninteracting system) in 1D systems has been demonstrated [7]. Although still a matter of controversy, the interaction effects do not destroy the condensate immediately according to the Monte Carlo simulations performed at finite temperature of Pearson, Pang, and Chen [11]. A finite-temperature analysis (finding a nonzero critical temperature $T_{\mathrm{c}}$ ) is necessary to establish the occurrence of a true BEC. Our study was at zero temperature, thus, it does not address the transition to a Bose-Einstein condensed phase. The solutions of the 1D-GP equation, however, indicate the formation of spatial distribution of bosons of a finite size which may be construed as a condensate cloud. We have used $\eta=3 / 2$ in our numerical examples, but the prescribed method applies to more general values of $\eta<2$. Furthermore, a similar analysis appears to be possible for harmonic traps in 1D to model the cigar-shaped condensates with suitable averaging over the cross-section and identifying the renormalized parameters as discussed by various authors [9]. Another important issue to be addressed is the effect of quantum fluctuations [4] on the interacting boson system. It was found in quasi-two-dimensional systems [26] that phase fluctuations are small at sufficiently low temperatures.

In summary, we have considered the ground-state properties of $1 \mathrm{D}$ condensates in power law trapping potentials at zero temperature. The corrections to the condensate wave function are evaluated within a perturbation approach for various coupling strengths and particle number. Using the exact solution of the 1D homogeneous system of interacting bosons, we construct a new nonlinear equation to describe the condensate wave function in the strongcoupling regime. We have shown at the Thomas-Fermi approximation level that the infinitely strongly interacting bosons are equivalent to noninteracting spinless fermions which is a manifestation of Girardeau's theorem. It would be interesting to study the further properties of the new nonlinear equation such as the vortex solutions and the details of the collective excitations, and explore the boson-fermion equivalence mentioned above. The fate of the condensate at finite temperature and quantum fluctuations also remains to be investigated.

$$
* * *
$$

This work was partially supported by the Scientific and Technical Research Council of Turkey (TUBITAK) under Grant No. TBAG-1662, by NATO under Grant No. SfP971970, and by the Turkish Department of Defense under Grant No. KOBRA-001. We thank Dr. M. Z. GEDIK for useful discussions. We are indebted to Dr. W. KRAuth for providing us with his GP equation solving code. 


\section{REFERENCES}

[1] Anderson M. H., Ensher J. R., Matthews M. R., Wieman C. E. and Cornell E. A., Science, 269 (1995) 198; Davis K. B., Mewes M.-O., Andrews M. R., van Druten N. J., Durfee D. S., Kurn D. M. and Ketterle W., Phys. Rev. Lett., 75 (1995) 3969; Bradley C. C., Sackett C. A. and Hulet R. G., Phys. Rev. Lett., 78 (1997) 985.

[2] Dalfovo F., Giorgini S., Pitaevskit L. and Stringari S., Rev. Mod. Phys., 71 (1999) 463; Parkins A. S. and Walls D. F., Phys. Rep., 303 (1998) 1.

[3] Timmermans E., Tommasini P. and Huang K., Phys. Rev. A, 55 (1997) 3645.

[4] Braten E. and Nieto A., Phys. Rev. B, 56 (1997) 14745.

[5] Fabrocini A. and Polls A., Phys. Rev. A, 60 (1999) 2319.

[6] Nunes G. S., J. Phys. B, 32 (1999) 4293.

[7] Ketterle W. and van Druten N. J., Phys. Rev. A, 54 (1996) 656; Bayindir M., Tanatar B. and Gedik Z., Phys. Rev. A, 59 (1999) 1468; for an early work see De Groot S. R., Hooyman G. J. and ten Seldam C. A., Proc. R. Soc. London, Ser. A, 203 (1950) 266.

[8] Pinkse P. W. H., Mosk A., Weidenmüller M., Reynolds M. W., Hijmans T. W. and Walraven J. T. M., Phys. Rev. Lett., 78 (1997) 990.

[9] Pérez-García V. M., Michinel H. and Herrero H., Phys. Rev. A, 57 (1998) 3837; Kivshar Y. S. and Alexander T. J., preprint cond-mat/9905048.

[10] Haugset T. and Haugerud H., Phys. Rev. A, 57 (1998) 3809; Mullin W. J., J. Low Temp. Phys., 110 (1998) 167; Monien H., Linn M. and Elstner N., Phys. Rev. A, 58 (1998) 3395; Olshanit M., Phys. Rev. Lett., 81 (1998) 938.

[11] Pearson S., Pang T. and Chen C., Phys. Rev. A, 58 (1998) 1485.

[12] Gross E. P., Nuovo Cimento, 20 (1961) 454; PitaevskiI L. P., Zh. Eksp. Teor. Fiz., 40 (1961) 646 (Sov. Phys. JETP, 13 (1961) 451).

[13] Lieb E. H., Seiringer R. and Yngvason J., Phys. Rev. A, 61 (2000) 3602; preprint condmat/0005026.

[14] Lieb E. H. and Liniger W., Phys. Rev., 130 (1963) 1605.

[15] Baym G. and Pethick C., Phys. Rev. B, 76 (1996) 6.

[16] Kohn W. and Sham L. J., Phys. Rev., 140 (1965) 1133.

[17] Huang K. and Yang C. N., Phys. Rev., 105 (1957) 767; Lee T. D. and Yang C. N., Phys. Rev., 106 (1957) 1119; Lee T. D., Huang K. and Yang C. N., Phys. Rev., 106 (1957) 1135.

[18] Gold A., Z. Phys. B, 91 (1993) 397; Demirel E. and Tanatar B., Phys. Rev. B, 59 (1999) 9271.

[19] Girardeau M., J. Math. Phys., 1 (1960) 516.

[20] Butts D. A. and Rokhsar D. S., Phys. Rev. A, 55 (1997) 4346.

[21] Ziegler K. and Shukla A., Phys. Rev. A, 57 (1998) 1464.

[22] Cherny A. Yu. and Shanenko A. A., preprint cond-mat/9904217.

[23] Gammal A., Frederico T., Tomio L. and Chomaz Ph., Phys. Rev. A, 61 (2000) 1602.

[24] Giorgini S., Boronat J. and Casulleras J., Phys. Rev. A, 60 (1999) 5129.

[25] Stringari S., Phys. Rev. Lett., 77 (1996) 2360; Pitaevskit L. and Stringari S., Phys. Rev. Lett., 81 (1998) 4541.

[26] Petrov D. S., Holzmann M. and Shlyapnikov G. V., Phys. Rev. Lett., 84 (2000) 2551. 